\title{
Fluorine F 18 Galacto-RGD Peptide
}

National Cancer Institute

\section{Source}

National Cancer Institute. Fluorine F18 Galacto-RGD Peptide. NCI Thesaurus. Code C112178.

A radiotracer composed of a cyclic Arg-Gly-Asp (RGD) peptide that is conjugated with galactose and radiolabeled with fluorine 18 , for potential noninvasive positron emission tomography (PET) imaging of alphaVbeta3 integ rin expression. Upon administration, the RGD moiety of the fluorine 18 galacto-RGD peptide selectively binds to alphaVbeta3 integrin. During PET imaging, alphaVbeta3-expressing tumor cells can be visualized and the degree of tumor angiogenesis can be determined. Use of a hydrophilic glycopeptidebased agent may increase retention in the blood and reduce clearance by the liver. This leads to both increased uptake of fluorine 18 galacto-RGD peptide by tumor cells and enhanced PET imaging efficiency, when compared to other agents that are constructed using lipophilic conjugates. AlphaVbeta3 integrin, a cell adhesion and signaling receptor, is upregulated in tumor vessel endothelial cells and has been associated with proliferation of tumor cells, tumor angiogenesis and metastasis. 